Results Data between the Warfarin, Rivaroxaban and Edoxaban groups were analysed using a multivariate analysis. Warfarin was used as the reference group with age and gender as covariates. Acute thromboembolic, bleeding and other less common complications were compared (table 2). There were 4 complications $(4.6 \%)$ in the warfarin, $2(2.5 \%)$ in the Rivaroxaban and $2(3 \%)$ in the Edoxaban groups, respectively. ( $\mathrm{P}$ value $=0.9)$.

Conclusion This retrospective study demonstrated that uninterrupted Edoxaban is as safe and effective as warfarin and Rivaroxaban in patients undergoing atrial fibrillation/ flutter ablations. There was no significant difference in acute bleeding and thromboembolic complications.

\section{IMPACT OF ATRIAL FIBRILLATION TERMINATION ON LONG TERM OUTCOME IN PERSISTENT ATRIAL FIBRILLATION PATIENTS UNDERGOING ACQMAP GUIDED ABLATION}

${ }^{1}$ Michael Pope*, ${ }^{2}$ Rui Shi, ${ }^{3}$ Mark Taylor, ${ }^{3}$ Moloy Das, ${ }^{3}$ Stephen Murray, ${ }^{4} T o m$ Wong ${ }^{1}$ Timothy Betts. ${ }^{1}$ Oxford University Hospitals NHS Foundation Trust; ${ }^{2}$ Royal Brompton and Harefield NHS Foundation Trust; ${ }^{3}$ Freeman Hospital, Newcastle; ${ }^{4}$ Brompton and Harefield NHS Foundation Trust

10.1136/heartjnl-2019-BCS.34

Introduction An acute response to ablation (arrhythmia termination or cycle length prolongation) is often presented as evidence of the significance of non-pulmonary vein (PV) mechanisms for AF maintenance and is an attractive procedural end point.

Objectives To investigate whether acute AF termination with ablation predicts long-term freedom from arrhythmia recurrence.

Methods We conducted a retrospective analysis of 100 patients who have undergone AcQMap (Acutus Medical) guided catheter ablation for AF at 3 UK centres. Acute success was defined as a spontaneous conversion to sinus rhythm (SR) during the ablation procedure. Long-term success was defined as freedom from any documented arrhythmia after a 3-month blanking period.

Results Mean follow up was $11 \pm 4$ months. Baseline characteristics were well matched between groups except that a higher proportion of those with acute success were in SR at baseline $(\mathrm{n}=11,28 \%$, vs. $\mathrm{n}=6,10 \%, \mathrm{p}=0.022)$.

During follow up, 68\% (27/40) of patients with acute termination were free of arrhythmia recurrence compared with $50 \%(30 / 60)$ of those who required DCCV, a non-statistically significant difference of $0.18, p=0.083$. Binomial logistic regression was performed to evaluate the combined effect of acute termination and baseline SR on long-term outcome. The logistic regression model was not statistically significant $(\chi 2=3.857, \mathrm{p}=0.145)$. For those undergoing first time procedures $(n=81)$ ablation to SR vs DCCV freedom from recurrent arrhythmia was $74 \%$ vs $51 \%$ $(p=0.041)$. In those in AF at baseline $(n=83)$ ablation to SR vs DCCV freedom from recurrent arrhythmia was $69 \%$ vs $56 \%(p=0.048)$.

Conclusion The relationship between acute ablation to SR and long-term outcome is unclear but may be important for certain subgroups. More work is needed to fully explore the significance of this endpoint.

Conflict of Interest None

\section{YOU WANT MY ADVICE AND GUIDANCE? PERFORMANCE OF A CARDIOLOGY EMAIL ADVICE LINE TO GENERAL PRACTITIONERS}

'John Belsham*, 'David G Wilson, 'Will Foster, 'Jasper Trevelyan, 'Bashar Aldhoon, ${ }^{1}$ David Smith, 'Deepak Goyal, 'William Roberts, ${ }^{1}$ Francesco Formisano, ${ }^{1}$ Robin Taylor, ${ }^{1}$ Charlotte Miles, ${ }^{2}$ John Tudor, ${ }^{1}$ Simon Gates, ${ }^{1}$ Helen Routledge. ${ }^{1}$ Worcestershire Acute Hospitals NHS Trust; ${ }^{2}$ NHS Wyre Forest CCG

\subsection{6/heartjnl-2019-BCS.35}

Background Cardiologists at Worcestershire Royal Hospital have provided email advice and guidance for local general practitioners. The performance of this service has not been formally evaluated.

Methodology Data were collected prospectively throughout 2018 including patient demographics, GP practice, time/date of email and of response, the person responding, the nature of the clinical question and whether admission or clinic appointment was recommended. Patient records were reviewed two months after the email to evaluate outcome.

Results In 2018, 2157 email queries were received about patients with a median age of 68 years (IQR 53-77). 52\% were male.

In total 2145 queries (99.44\%) were sent on weekdays, mean of 8.25 per weekday. The median response time was 4 hours 19 minutes (IQR 1.78-17.45 hours), 14.6\% received a response in under an hour, $86.1 \%$ of queries were responded to within 24 hours and $93.9 \%$ within 48 hours. Weekend emails explained the remainder.

Overall $91.4 \%$ of emails were received between $0800-1700$ and $36.2 \%$ of responses were sent outside of this time. We estimate 215 consultant hours were taken replying (assuming 6 minutes/email) equivalent to 54 programmed activity (PA) episodes.

The most common queries related to interpretation or management of ECGs (44\%), ambulatory ECG monitoring $(12.5 \%)$ or echocardiography $(9.2 \%)$.

In total 73 GP Practices sent emails (mean of 28.6 queries/ practice) of which $63(86.3 \%)$ were from the Worcestershire CCG area. Worcestershire GPs accounted for $97.5 \%$ of all queries.

Variation exists between individual practices: three did not send any email queries; seven sent only one query over the year and one practice sent 179 queries (8.6\% of total). If all practices participated as frequently, the number of emails received would increase five-fold to over 10,000 a year.

Of the 10 Cardiology consultants within the deparment, not all participated equally with three consultants replying to $63 \%$ of all emails and one replying to $30 \%$.

Admission to hospital was recommended in one case and referral to outpatient clinic was recommended in 501 of 2152 cases $(23.2 \%)$. Of these, a referral was only received within two months in $60.6 \%$ of cases.

We estimate that this initiative avoided up to 1493 clinic appointments (124 Consultant PA episodes or 2 per week).

Potential cost savings to CCG $(1493 \times £ 168$ for cost of new outpatient consultation) $=£ 250,824$. Potential revenue to cardiology for ECG interpretation (44\% of $2153 \times £ 25=$ $£ 23,683)$ and for 24 hour holter interpretation $12.5 \%$ of $2153 \times £ 45=£ 12,110)$

(Price estimate from NICE Remote ECG interpretation consultancy services for cardiovascular disease MIB152))

Conclusion A cardiology advice and guidance email service is highly efficient use of specialist consultant time with a large 
number of outpatient clinic appointments being avoided at a cost saving to the CCG.

Conflict of Interest None

\section{EFFICACY OF DC CARDIOVERSION FOR ATRIAL FIBRILLATION: A LARGE RETROSPECTIVE OBSERVATIONAL STUDY}

Alexander Carpenter*, John Graby, Rachael Medland, Stewart Brown, Claire Sowerby, Louise Priestman, Mark Dayer, Guy Furniss. Taunton and Somerset NHS Foundation Trust

\subsection{6/heartjnl-2019-BCS.36}

Introduction The evidence base for rhythm control strategies in atrial fibrillation (AF) remain contentious. Synchronised DC cardioversion (DCCV) is a simple and accessible treatment option in many centres. However, there is little data to support its use as a long-term strategy or data regarding which groups benefit most from this strategy.

Methods We collected AF cardioversion cases across a six-year period at a busy district general hospital with a nurse-led cardioversion service. Demographic, echocardiographic and procedural data were collected, as were safety and AF outcomes at 6 and 12 months. Patients with incomplete data were excluded from the analysis. Statistical analysis was undertaken using the Fischer's exact T-test.

Results 550 cardioversion cases were included in the analysis with a median age of 67 (range 28-95). 163 (30\%) of patients were obese (BMI 30). The mean CHA2DS2-VASc was 2.3. $342(62 \%)$ of DCCV were for persistent AF, the remainder $(208 ; 38 \%)$ longstanding (AF duration $>1$ year) persistent. $162(29 \%)$ had an unknown duration of AF due to an incidental diagnosis. $483(88 \%)$ were on AV-nodal blocking and 103 (19\%) on anti-arrhythmic medication (flecainide, sotalol or amiodarone). 516 (94\%) of DCCVs were acutely successful with a complication rate of $2 \%(n=13)$, most commonly symptomatic bradycardia requiring temporary transcutaneous $(n=2)$ or percussion pacing $(n=6)$ with no acute strokes. At 1 year $5(0.9 \%)$ had had a stroke/transient ischaemic attack and $9(1.6 \%)$ died. 179/478 (37\%) of patients were free from AF at 6 months, with $100 / 412$ $(24 \%)$ free from AF at 1 year. 89 (16\%) of patients were referred for further cardioversion and 144 (26\%) referred for catheter ablation. There was no statistically significant effect of patient age, obesity, left ventricular (LV) impairment, or left atrial (LA) dilatation on 6-month outcomes. However, AF duration $<1$ year did correlate with statistically significant improvement in 6 month freedom from AF $(41 \%$ vs $31 \%, p=0.03)$. In those with moderate/severe LV impairment, anti-arrhythmic use significantly improved 6month outcomes $(70 \%$ vs $40 \%, p=0.01)$. However, no such effect was seen between moderate/severely dilated and nondilated LA.

Conclusion DCCV for AF remains a safe procedure with good acute success rates. However, within 6 months the majority of patients will have reverted to AF. AF duration of less than 1 year is associated with an improved 6-month success rate, as does antiarrhythmic use in those with impaired LV function. In selected patients, DCCV remains a useful tool as a 'trial of sinus rhythm' to establish potential symptomatic benefits from pursuing a rhythm control strategy.

Conflict of Interest Nil

\section{INVESTIGATION OF INTERMITTENT PALPITATIONS WITH THE MICOR ECG WRISTBAND}

Birkhoelzer Sarah*, N Mullan, J Crombie, P Archer, J Gillings, Mark A Tanner. St Richard's Hospital

\subsection{6/heartjnl-2019-BCS.37}

Background Palpitations are a leading cause of referral to cardiology services. The diagnosis of arrhythmia is achieved through establishing a symptom-rhythm correlation. Conventional ambulatory ECG monitoring can fail to capture symptomatic events.

Objective The ECG wristband is a wearable patient-activated lead I ECG recorder (30 seconds per event) permitting longterm monitoring and might offer a useful strategy for the initial investigation of palpitations.

Methods Patients referred to our service for investigation of paroxysmal arrhythmia were considered suitable for use of the MiCor device if they reported palpitations (or arrhythmic symptoms e.g. dizzy spells). Patients with symptoms of syncope or inability to use the device were excluded. Patients were

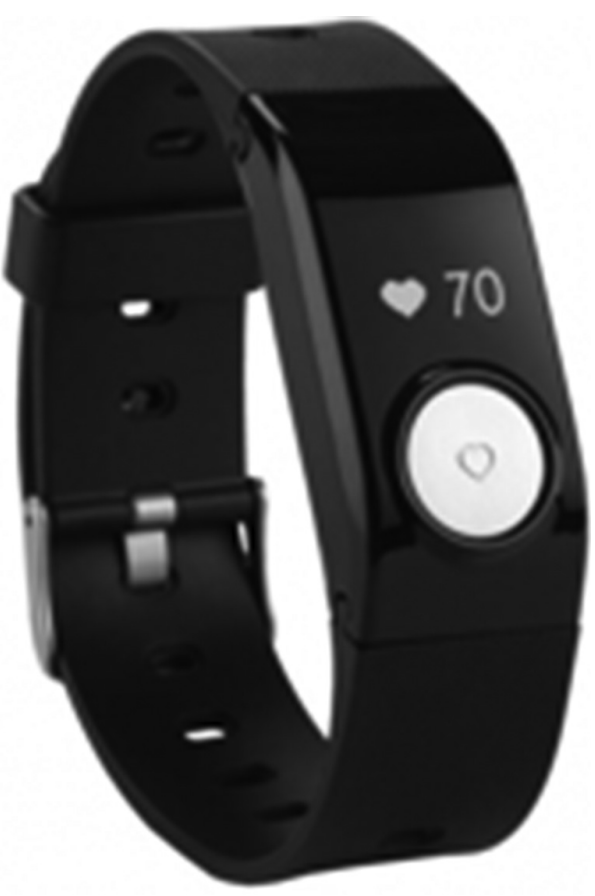

Abstract 39 Figure 1 Micor A100 wristband

Abstract 39 Table 1 Symptom-rhythm correlation $37 / 38$ patients with symptom-rhythm correlation

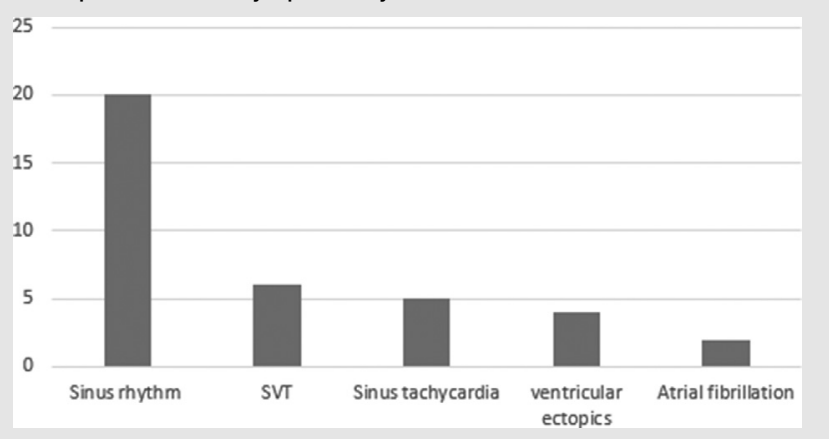

